\title{
Sums of Averages of GCD-Sum Functions II
}

\author{
Lisa Kaltenböck, Isao Kiuchi, Sumaia Saad Eddin®, and \\ Masaaki Ueda
}

\begin{abstract}
Let $\operatorname{gcd}(k, j)$ denote the greatest common divisor of the integers $k$ and $j$, and let $r$ be any fixed positive integer. Define

$$
M_{r}(x ; f):=\sum_{k \leq x} \frac{1}{k^{r+1}} \sum_{j=1}^{k} j^{r} f(\operatorname{gcd}(j, k))
$$

for any large real number $x \geq 5$, where $f$ is any arithmetical function. Let $\phi$, and $\psi$ denote the Euler totient and the Dedekind function, respectively. In this paper, we refine asymptotic expansions of $M_{r}(x ;$ id), $M_{r}(x ; \phi)$ and $M_{r}(x ; \psi)$. Furthermore, under the Riemann Hypothesis and the simplicity of zeros of the Riemann zeta-function, we establish the asymptotic formula of $M_{r}(x$; id) for any large positive number $x>5$ satisfying $x=[x]+\frac{1}{2}$.
\end{abstract}

Mathematics Subject Classification. 11A25, 11 N37.

Keywords. GCD-sum functions, the Euler totient function, the Dedekind function, the Dirichlet divisor problem, The Riemann Hypothesis, Simple zeros of the Riemann zeta-function.

\section{Introduction and Statement of Results}

Let $\operatorname{gcd}(k, j)$ be the greatest common divisor of the integers $k$ and $j$. The gcdsum function, which is also known as Pillai's arithmetical function, is defined

Lisa Kaltenböck is supported by the Austrian Science Fund (FWF), Project F5507-N26, which is a part of the Special Research Program "Quasi Monte Carlo Methods: Theory and Applications". Sumaia Saad Eddin is supported by the Austrian Science Fund (FWF): Projects F5507-N26 and F5505-N26, which are parts of the Special Research Program "Quasi Monte Carlo Methods: Theory and Applications" . 
by

$$
P(n)=\sum_{k=1}^{n} \operatorname{gcd}(k, n) .
$$

This function has been studied by many authors such as Broughan [4], Bordellés [3], Tanigawa and Zhai [18], Tóth [19], and others. Analytic properties for partial sums of the gcd-sum function $f(\operatorname{gcd}(j, k))$ were recently studied by Inoue and Kiuchi [8]. We recall that the symbol $*$ denotes the Dirichlet convolution of two arithmetical functions $f$ and $g$ defined by $f * g(n)=$ $\sum_{d \mid n} f(d) g(n / d)$, for every positive integer $n$. For any arithmetical function $f$, the second author [11] showed, that for any fixed positive integer $r$ and any large positive number $x \geq 2$ we have

$$
\begin{aligned}
M_{r}(x ; f):= & \sum_{k \leq x} \frac{1}{k^{r+1}} \sum_{j=1}^{k} j^{r} f(\operatorname{gcd}(k, j)) \\
= & \frac{1}{2} \sum_{n \leq x} \frac{f(n)}{n}+\frac{1}{r+1} \sum_{d \ell \leq x} \frac{\mu * f(d)}{d} \\
& +\frac{1}{r+1} \sum_{m=1}^{r / 2]}\left(\begin{array}{c}
r+1 \\
2 m
\end{array}\right) B_{2 m} \sum_{d \ell \leq x} \frac{\mu * f(d)}{d} \frac{1}{\ell^{2 m}} .
\end{aligned}
$$

Here, as usual, the function $\mu$ denotes the Möbius function and $B_{m}=B_{m}(0)$ are the Bernoulli numbers, with $B_{m}(x)$ being the Bernoulli polynomials defined by the generating function

$$
\frac{z e^{x z}}{e^{z}-1}=\sum_{m=0}^{\infty} B_{m}(x) \frac{z^{m}}{m !}
$$

with $|z|<2 \pi$. Many applications of Eq. (1) have been given in [10], [12] and [13].

In [11], Eq. (1) was used to establish asymptotic formulas for $M_{r}(x ; f)$ for specific choices of $f$ such as the identity function id, the Euler totient function $\phi=\mathrm{id} * \mu$ or the Dedekind function $\psi=\mathrm{id} *|\mu|$. More precisely, let $\zeta(s)$ denote the Riemann zeta-function, then for $f=\mathrm{id}$, it was proved that

$$
\begin{aligned}
M_{r}(x ; \text { id })= & \frac{1}{(r+1) \zeta(2)} x \log x+\frac{x}{2} \\
& +\frac{1}{(r+1) \zeta(2)}\left(2 \gamma-1-\frac{\zeta^{\prime}(2)}{\zeta(2)}+\sum_{m=1}^{[r / 2]}\left(\begin{array}{c}
r+1 \\
2 m
\end{array}\right) B_{2 m} \zeta(2 m+1)\right) \\
& x+K_{r}(x),
\end{aligned}
$$

where

$$
K_{r}(x)=\frac{1}{r+1} \sum_{n \leq x} \frac{\mu(n)}{n} \Delta\left(\frac{x}{n}\right)+O_{r}(\log x) .
$$


For $f=\phi$, it was shown that

$$
\begin{aligned}
M_{r}(x ; \phi)= & \frac{1}{(r+1) \zeta^{2}(2)} x \log x+\frac{x}{2 \zeta(2)} \\
& +\frac{1}{(r+1) \zeta^{2}(2)}\left(2 \gamma-1-2 \frac{\zeta^{\prime}(2)}{\zeta(2)}+\sum_{m=1}^{[r / 2]}\left(\begin{array}{c}
r+1 \\
2 m
\end{array}\right) B_{2 m} \zeta(2 m+1)\right) \\
& x+L_{r}(x)
\end{aligned}
$$

where

$$
L_{r}(x):=\frac{1}{r+1} \sum_{n \leq x} \frac{\mu * \mu(n)}{n} \Delta\left(\frac{x}{n}\right)+O_{r}\left((\log x)^{2}\right) .
$$

Lastly, for $f=\psi$ it was proved that

$$
\begin{aligned}
& M_{r}(x ; \psi)=\frac{1}{(r+1) \zeta(4)} x \log x+\frac{\zeta(2)}{2 \zeta(4)} x \\
& \quad+\frac{1}{(r+1) \zeta(4)}\left(2 \gamma-1-2 \frac{\zeta^{\prime}(4)}{\zeta(4)}+\sum_{m=1}^{[r / 2]}\left(\begin{array}{c}
r+1 \\
2 m
\end{array}\right) B_{2 m} \zeta(2 m+1)\right) x+U_{r}(x),
\end{aligned}
$$

where

$$
U_{r}(x):=\frac{1}{r+1} \sum_{n \leq x} \frac{\mu *|\mu|(n)}{n} \Delta\left(\frac{x}{n}\right)+O_{r}\left((\log x)^{2}\right) .
$$

The function $\Delta(x)$ denotes the error term of the Dirichlet divisor problem: Let $\tau=\mathbf{1} * \mathbf{1}$ be the divisor function, then for any large positive number $x \geq 2$,

$$
\sum_{n \leq x} \tau(n)=x \log x+(2 \gamma-1) x+\Delta(x),
$$

where $\gamma$ is the Euler constant and $\Delta(x)$ can be estimated by $\Delta(x)=O\left(x^{\theta+\varepsilon}\right)$. It is known that one can take $1 / 4 \leq \theta \leq 1 / 3$. More precisely, the Dirichlet divisor problem is to find the smallest value of $\theta$ for which the above estimate holds, for any $\epsilon>0$. This problem is still unsolved. The best estimate to date is

$$
O\left(x^{131 / 416}(\log x)^{26947 / 8320}\right),
$$

obtained by Huxley [7] in 2003.

The first purpose of this paper is to refine the error terms $K_{r}(x), L_{r}(x)$ and $U_{r}(x)$ from the above formulas. Therefore, let $\sigma_{u}=\mathrm{id}_{u} * \mathbf{1}$ be the generalized divisor function for any real number $u$ and let $m \geq 1$ be an integer. Then for any large positive number $x \geq 2$, the function $\Delta_{-2 m}(x)$ denotes the error term of the generalized divisor problem given by

$$
\sum_{n \leq x} \sigma_{-2 m}(n)=\zeta(1+2 m) x-\frac{1}{2} \zeta(2 m)+\Delta_{-2 m}(x) .
$$


For more details about the functions $\Delta(x), \Delta_{-2 m}(x)$, see [1]. We have the following results:

Theorem 1. Let $\Delta(x)$ and $\Delta_{-2 m}(x)$ be the error terms given by Eqs. (5) and (6), respectively. For any large positive number $x>5$ and fixed positive integer $r$, we have

$$
\begin{aligned}
K_{r}(x)= & \frac{1}{r+1} \sum_{d \leq x} \frac{\mu(d)}{d} \Delta\left(\frac{x}{d}\right) \\
& +\frac{1}{r+1} \sum_{d \leq x} \frac{\mu(d)}{d} \sum_{m=1}^{[r / 2]}\left(\begin{array}{c}
r+1 \\
2 m
\end{array}\right) B_{2 m} \Delta_{-2 m}\left(\frac{x}{d}\right)+O_{r}(\delta(x)),
\end{aligned}
$$

where the function $\delta(x)$ is defined by

$$
\delta(x):=\exp \left(-C \frac{(\log x)^{3 / 5}}{(\log \log x)^{1 / 5}}\right)
$$

with $C$ being a positive constant. Moreover, we have

$$
\begin{aligned}
L_{r}(x)= & \frac{1}{r+1} \sum_{n \leq x} \frac{\mu * \mu(n)}{n} \Delta\left(\frac{x}{n}\right) \\
& +\frac{1}{r+1} \sum_{n \leq x} \frac{\mu * \mu(n)}{n} \sum_{m=1}^{[r / 2]}\left(\begin{array}{c}
r+1 \\
2 m
\end{array}\right) B_{2 m} \Delta_{-2 m}\left(\frac{x}{n}\right) \\
& +O_{r}\left((\log x)^{2 / 3}(\log \log x)^{1 / 3}\right) .
\end{aligned}
$$

and

$$
\begin{aligned}
U_{r}(x)= & \frac{1}{r+1} \sum_{n \leq x} \frac{\mu *|\mu|(n)}{n} \Delta\left(\frac{x}{n}\right) \\
& +\frac{1}{r+1} \sum_{n \leq x} \frac{\mu *|\mu|(n)}{n} \sum_{m=1}^{[r / 2]}\left(\begin{array}{c}
r+1 \\
2 m
\end{array}\right) B_{2 m} \Delta_{-2 m}\left(\frac{x}{n}\right) \\
& -\frac{1}{4 \zeta(2)} \log x+O_{r}\left((\log x)^{2 / 3}\right) .
\end{aligned}
$$

Remark 1. It is easily checked that using the weakest estimate $\Delta_{-2 m}(x)=$ $O_{m}(1)$ in the results Theorem 1 yields much better results than the previously known formulas for $K_{r}(x), L_{r}(x)$ and $M_{r}(x)$ from Eqs. (2), (3), and (4).

Furthermore, even better estimates of $K_{r}(x)$ can be achieved by additional assumptions on the Riemann zeta-function. Under the Riemann Hypothesis, Maier and Montgomery [15] gave a sharper estimate of the partial sum of the Möbius function, which was later improved by Soundararajan [17]. 
The author proved that

$$
M(x):=\sum_{n \leq x} \mu(n)=O\left(x^{1 / 2} \eta(x)\right)
$$

where

$$
\eta(x):=\exp \left((\log x)^{1 / 2}(\log \log x)^{14}\right),
$$

for any large positive number $x>5$ satisfying $x=[x]+\frac{1}{2}$. This latter has been improved slightly by Balazard and de Roton [2]. By using the above result on $M(x)$, we obtain the next statement.

Theorem 2. Assume the Riemann Hypothesis and let $\Delta(x)$ and $\Delta_{-2 m}(x)$ be the error terms given by Eqs. (5) and (6), respectively. Then for any large positive number $x>5$ such that $x=[x]+\frac{1}{2}$ and fixed positive integer $r$, we have

$$
\begin{aligned}
K_{r}(x)= & \frac{1}{r+1} \sum_{d \leq x} \frac{\mu(d)}{d} \Delta\left(\frac{x}{d}\right) \\
& +\frac{1}{r+1} \sum_{d \leq x} \frac{\mu(d)}{d} \sum_{m=1}^{[r / 2]}\left(\begin{array}{c}
r+1 \\
2 m
\end{array}\right) B_{2 m} \Delta_{-2 m}\left(\frac{x}{d}\right)+O_{r}\left(\frac{\eta(x) \log x}{x^{1 / 2}}\right) .
\end{aligned}
$$

For our further considerations, let $\rho=\alpha+i \beta$ denote the generic nontrivial zeros of the Riemann zeta-function. Under the assumption that all zeros $\rho$ in the critical strip of $\zeta(s)$ are simple, we are able to prove an additional refinement for the error term $K_{r}(x)$.

Theorem 3. Assume that the zeros of $\zeta(s)$ are simple. Let $T_{*} \geq x^{6}$ be some positive number satisfying the inequality

$$
\frac{1}{\zeta\left(\sigma+i T_{*}\right)} \ll T_{*}^{\varepsilon}
$$

for $\frac{1}{2} \leq \sigma \leq 2$. For any large positive number $x>5$ with $x=[x]+\frac{1}{2}$ we then have

$$
\begin{aligned}
K_{r}(x)= & \frac{1}{r+1} \sum_{n \leq x} \frac{\mu(n)}{n} \Delta\left(\frac{x}{n}\right)+\frac{1}{r+1} \sum_{n \leq x} \frac{\mu(n)}{n} \sum_{m=1}^{[r / 2]}\left(\begin{array}{c}
r+1 \\
2 m
\end{array}\right) B_{2 m} \Delta_{-2 m}\left(\frac{x}{n}\right) \\
& +\frac{2 \gamma+C_{\text {odd }}(r)-1}{r+1} \sum_{|\beta| \leq T_{*}} \frac{x^{\rho-1}}{(\rho-2) \zeta^{\prime}(\rho)}-\frac{C_{\text {even }}(r)}{2(r+1)} \sum_{|\beta| \leq T_{*}} \frac{x^{\rho-1}}{(\rho-1) \zeta^{\prime}(\rho)} \\
& +\frac{1}{r+1} \sum_{|\beta| \leq T_{*}} \frac{x^{\rho-1}}{(\rho-2)^{2} \zeta^{\prime}(\rho)}+O_{r}\left(x^{-3}\right)
\end{aligned}
$$


where the functions $C_{\text {odd }}(r)$ and $C_{\text {even }}(r)$ are given by

$$
C_{\text {odd }}(r):=\sum_{m=1}^{[r / 2]}\left(\begin{array}{c}
r+1 \\
2 m
\end{array}\right) B_{2 m} \zeta(2 m+1),
$$

and

$$
C_{\text {even }}(r):=\sum_{m=1}^{[r / 2]}\left(\begin{array}{c}
r+1 \\
2 m
\end{array}\right) B_{2 m} \zeta(2 m)
$$

for any fixed positive integer $r$.

Finally, define the sum

$$
J_{-\lambda}(T):=\sum_{0<\beta \leq T} \frac{1}{\left|\zeta^{\prime}(\rho)\right|^{2 \lambda}}
$$

which is intimately connected to Mertens function. Assuming the simplicity of the zeros of $\zeta(s)$, Gonek [5] and Hejhal [6] independently conjectured that for any real number $\lambda<3 / 2$, we have

$$
J_{-\lambda}(T) \asymp T(\log T)^{(\lambda-1)^{2}}
$$

We use this conjecture to prove the following:

Theorem 4. Assume that the Riemann Hypothesis and Gonek-Hejhal conjecture. Then

$$
\begin{aligned}
K_{r}(x)= & \frac{1}{r+1} \sum_{n \leq x} \frac{\mu(n)}{n} \Delta\left(\frac{x}{n}\right)+\frac{1}{r+1} \sum_{d \leq x} \frac{\mu(d)}{d} \sum_{m=1}^{[r / 2]}\left(\begin{array}{c}
r+1 \\
2 m
\end{array}\right) B_{2 m} \Delta_{-2 m}\left(\frac{x}{d}\right) \\
& +O_{r}\left(\frac{(\log x)^{5 / 4}}{x^{1 / 2}}\right),
\end{aligned}
$$

for any large positive number $x>5$ satisfying $x=[x]+\frac{1}{2}$.

\section{Proofs of Theorems 1 and 2}

In order to prove our main results, we first show some necessary lemmas.

\subsection{Auxiliary Lemmas}

Lemma 1. For any large positive number $x>5$, we have

$$
\begin{gathered}
\sum_{n \leq x} \frac{\mu(n)}{n^{2}}=\frac{1}{\zeta(2)}+O\left(\frac{\delta(x)}{x}\right), \\
\sum_{n \leq x} \frac{\mu(n)}{n^{2}} \log n=\frac{\zeta^{\prime}(2)}{\zeta^{2}(2)}+O\left(\frac{\delta(x)}{x}\right),
\end{gathered}
$$


and

$$
\sum_{n \leq x} \frac{\mu(n)}{n}=O(\delta(x)),
$$

where $\delta(x)$ is given by Eq. (7). Assume that $x=[x]+\frac{1}{2}$. Under the Riemann Hypothesis we have

$$
\begin{gathered}
\sum_{n \leq x} \frac{\mu(n)}{n^{2}}=\frac{1}{\zeta(2)}+O\left(\frac{\eta(x)}{x^{3 / 2}}\right), \\
\sum_{n \leq x} \frac{\mu(n)}{n^{2}} \log n=\frac{\zeta^{\prime}(2)}{\zeta^{2}(2)}+O\left(\frac{\eta(x) \log x}{x^{3 / 2}}\right),
\end{gathered}
$$

and

$$
\sum_{n \leq x} \frac{\mu(n)}{n}=O\left(\frac{\eta(x)}{x^{1 / 2}}\right) .
$$

for any large positive number $x>5$. Here $\eta(x)$ is given by Eq. (8).

Proof. Eqs. (10) and (11) follow from Lemmas 2.2 and 2.3 in [16]. The proof of Eq. (12) can be found in [9]. The formulas (13)-(15) follow from Lemma 2.1 in $[8]$.

Lemma 2. For any large positive number $x>5$, we have

$$
\sum_{n \leq x} \frac{\phi(n)}{n}=\frac{x}{\zeta(2)}+O\left((\log x)^{2 / 3}(\log \log x)^{1 / 3}\right) .
$$

Proof. For any large positive number $x \geq 5$, we use the result of Liu in [14]

$$
\sum_{\ell \leq x} \frac{\mu(\ell)}{\ell} \vartheta\left(\frac{x}{\ell}\right)=O\left((\log x)^{2 / 3}(\log \log x)^{1 / 3}\right),
$$

the fact that $\phi=\mathrm{id} * \mu$, and Eqs. (10), (12) to obtain the formula

$$
\begin{aligned}
\sum_{n \leq x} \frac{\phi(n)}{n} & =\sum_{\ell \leq x} \frac{\mu(\ell)}{\ell}\left(\frac{x}{\ell}-\vartheta\left(\frac{x}{\ell}\right)-\frac{1}{2}\right) \\
& =\frac{x}{\zeta(2)}+O\left((\log x)^{2 / 3}(\log \log x)^{1 / 3}\right) .
\end{aligned}
$$

Here $\vartheta(x)$ is the oscillatory function defined by $x-[x]-\frac{1}{2}$. This completes the proof.

Lemma 3. For any large positive number $x>5$, we have

$$
\sum_{n \leq x} \frac{\psi(n)}{n}=\frac{\zeta(2)}{\zeta(4)} x-\frac{1}{2 \zeta(2)} \log x+O\left((\log x)^{2 / 3}\right) .
$$

Proof. The proof can be found in [20, Satz 3]. 
Lemma 4. For any large positive number $x>5$, we have

$$
\begin{gathered}
\sum_{n \leq x} \frac{\mu * \mu(n)}{n^{2}}=\frac{1}{\zeta^{2}(2)}+O\left(\frac{\kappa(x)}{x}\right), \\
\sum_{n \leq x} \frac{\mu * \mu(n)}{n^{2}} \log n=2 \frac{\zeta^{\prime}(2)}{\zeta^{3}(2)}+O\left(\frac{\kappa(x)}{x}\right),
\end{gathered}
$$

and

$$
\sum_{n \leq x} \frac{\mu * \mu(n)}{n}=O(\kappa(x))
$$

where $\kappa(x)$ is given by

$$
\kappa(x)=\exp \left(-D(\log x \log \log x)^{1 / 3}\right)
$$

with $D$ being a positive constant.

Proof. Eqs. (16), (17) and (18) follow from Eqs. (3.5), (3.6) and (3.3) in [8], respectively.

Lemma 5. For any large positive number $x>5$, we have

$$
\begin{gathered}
\sum_{n \leq x} \frac{|\mu| * \mu(n)}{n^{2}}=\frac{1}{\zeta(4)}+O\left(\frac{\delta(x)}{x^{3 / 2}}\right), \\
\sum_{n \leq x} \frac{|\mu| * \mu(n)}{n^{2}} \log n=2 \frac{\zeta^{\prime}(4)}{\zeta^{2}(4)}+O\left(\frac{\delta(x)}{x^{3 / 2}}\right),
\end{gathered}
$$

and

$$
\sum_{n \leq x} \frac{|\mu| * \mu(n)}{n}=\frac{1}{\zeta(2)}+O\left(\frac{\delta(x)}{x^{1 / 2}}\right) .
$$

Proof. Eqs. (19) and (20) follow from Eqs. (3.7) and (3.8) in [8], respectively. It is known that

$$
\sum_{n=1}^{\infty} \frac{|\mu| * \mu(n)}{n}=\frac{1}{\zeta(2)},
$$

Now, we write our sums as follows

$$
\begin{aligned}
\sum_{n \leq x} \frac{|\mu| * \mu(n)}{n} & =\sum_{n=1}^{\infty} \frac{|\mu| * \mu(n)}{n}-\sum_{n>x} \frac{|\mu| * \mu(n)}{n} \\
& =\frac{1}{\zeta(2)}-\sum_{n>x} \frac{|\mu| * \mu(n)}{n} .
\end{aligned}
$$

To complete the proof, it remains to estimate the last sum above. Notice that

$$
\sum_{n>x} \frac{|\mu| * \mu(n)}{n}=\int_{x}^{\infty} \frac{\sum_{x<n \leq t}|\mu| * \mu(n)}{t^{2}} d t
$$


and that

$$
\sum_{n \leq x}|\mu| * \mu(n)=\sum_{n \leq \sqrt{x}} \mu(n)+O\left(x^{\epsilon}\right)=O\left(x^{1 / 2} \delta(x)\right),
$$

where we used Eq. (3.4) from [8]. Therefore, we have

$$
\sum_{n>x} \frac{|\mu| * \mu(n)}{n}=O\left(\int_{x}^{\infty} \frac{t^{1 / 2} \delta(t)}{t^{2}} d t\right)=O\left(\frac{\delta(x)}{x^{1 / 2}}\right),
$$

and Eq. (21) is proved.

Lemma 6. For any large positive number $x>5$, we have

$$
\begin{aligned}
\sum_{n \leq x} \sum_{d \mid n} \frac{\phi(d)}{d}= & \frac{1}{\zeta(2)} x \log x+\frac{1}{\zeta(2)}\left(2 \gamma-1-\frac{\zeta^{\prime}(2)}{\zeta(2)}\right) x \\
& +\sum_{d \leq x} \frac{\mu(d)}{d} \Delta\left(\frac{x}{d}\right)+O(\delta(x)),
\end{aligned}
$$

and

$$
\sum_{d \ell \leq x} \frac{\phi(d)}{d} \frac{1}{\ell^{2 m}}=\frac{\zeta(1+2 m)}{\zeta(2)} x+\sum_{d \leq x} \frac{\mu(d)}{d} \Delta_{-2 m}\left(\frac{x}{d}\right)+O_{m}(\delta(x))
$$

for any positive integer $m$. Suppose that $x=[x]+\frac{1}{2}$. Under the Riemann Hypothesis, we have

$$
\begin{aligned}
\sum_{n \leq x} \sum_{d \mid n} \frac{\phi(d)}{d}= & \frac{1}{\zeta(2)} x \log x+\frac{1}{\zeta(2)}\left(2 \gamma-1-\frac{\zeta^{\prime}(2)}{\zeta(2)}\right) x \\
& +\sum_{d \leq x} \frac{\mu(d)}{d} \Delta\left(\frac{x}{d}\right)+O\left(\frac{\eta(x) \log x}{x^{1 / 2}}\right),
\end{aligned}
$$

and

$$
\sum_{d \ell \leq x} \frac{\phi(d)}{d} \frac{1}{\ell^{2 m}}=\frac{\zeta(1+2 m)}{\zeta(2)} x+\sum_{d \leq x} \frac{\mu(d)}{d} \Delta_{-2 m}\left(\frac{x}{d}\right)+O_{m}\left(\frac{\eta(x)}{x^{1 / 2}}\right)
$$

Proof. We recall the identity $\frac{\phi}{\mathrm{id}} * \mathbf{1}=\frac{\mu}{\mathrm{id}} * \tau$. Using Eqs. (5), (10) and (11), we obtain

$$
\begin{aligned}
& \sum_{n \leq x} \sum_{d \mid n} \frac{\phi(d)}{d}=\sum_{d \leq x} \frac{\mu(d)}{d} \sum_{\ell \leq x / d} \tau(\ell) \\
& \quad=x(\log x+2 \gamma-1) \sum_{d \leq x} \frac{\mu(d)}{d^{2}}-x \sum_{d \leq x} \frac{\mu(d)}{d^{2}} \log d+\sum_{d \leq x} \frac{\mu(d)}{d} \Delta\left(\frac{x}{d}\right) \\
& \quad=\frac{1}{\zeta(2)} x \log x+\frac{1}{\zeta(2)}\left(2 \gamma-1-\frac{\zeta^{\prime}(2)}{\zeta(2)}\right) x+\sum_{d \leq x} \frac{\mu(d)}{d} \Delta\left(\frac{x}{d}\right)+O(\delta(x)),
\end{aligned}
$$


which completes the proof of Eq. (22). Further, we recall the identity $\frac{\phi}{\mathrm{id}} *$ $\mathrm{id}_{-2 m}=\frac{\mu}{\mathrm{id}} * \sigma_{-2 m}$, and use Eqs. (6), (10) and (12) to get

$$
\begin{aligned}
\sum_{d \ell \leq x} \frac{\phi(d)}{d} \frac{1}{\ell^{2 m}} & =\sum_{d \leq x} \frac{\mu(d)}{d} \sum_{\ell \leq x / d} \sigma_{-2 m}(\ell) \\
& =\sum_{d \leq x} \frac{\mu(d)}{d}\left(\zeta(1+2 m) \frac{x}{d}-\frac{1}{2} \zeta(2 m)+\Delta_{-2 m}\left(\frac{x}{d}\right)\right) \\
& =\frac{\zeta(1+2 m)}{\zeta(2)} x+\sum_{d \leq x} \frac{\mu(d)}{d} \Delta_{-2 m}\left(\frac{x}{d}\right)+O_{m}(\delta(x)) .
\end{aligned}
$$

This completes the proof of Eq. (23). Similarly, we use Eqs. (13), (14) and (15) to deduce Eqs. (24) and (25).

Lemma 7. For any large positive number $x>5$, we have

$$
\begin{aligned}
\sum_{n \leq x} \sum_{d \mid n} \frac{\mu * \phi(d)}{d}= & \frac{1}{\zeta^{2}(2)} x \log x+\frac{1}{\zeta^{2}(2)}\left(2 \gamma-1-2 \frac{\zeta^{\prime}(2)}{\zeta(2)}\right) x \\
& +\sum_{d \leq x} \frac{\mu * \mu(d)}{d} \Delta\left(\frac{x}{d}\right)+O(\kappa(x))
\end{aligned}
$$

and

$$
\sum_{d \ell \leq x} \frac{\mu * \phi(d)}{d} \frac{1}{\ell^{2 m}}=\frac{\zeta(1+2 m)}{\zeta^{2}(2)} x+\sum_{d \leq x} \frac{\mu * \mu(d)}{d} \Delta_{-2 m}\left(\frac{x}{d}\right)+O_{m}(\kappa(x))
$$

for any positive integer $m$. Here $\kappa(x)$ is defined above in Lemma 4.

Proof. We use the identity $\frac{\mu * \phi}{\mathrm{id}} * \mathbf{1}=\frac{\mu * \mu}{\mathrm{id}} * \tau$, Eqs. (5), (16), and (17) to obtain

$$
\begin{aligned}
& \sum_{k \leq x} \sum_{d \mid k} \frac{\mu * \phi(d)}{d}=\sum_{d \leq x} \frac{\mu * \mu(d)}{d} \sum_{\ell \leq x / d} \tau(\ell) \\
& \quad=x(\log x+2 \gamma-1) \sum_{d \leq x} \frac{\mu * \mu(d)}{d^{2}}-x \sum_{d \leq x} \frac{\mu * \mu(d)}{d^{2}} \log d+\sum_{d \leq x} \frac{\mu * \mu(d)}{d} \Delta\left(\frac{x}{d}\right) \\
& \quad=\frac{x \log x}{\zeta^{2}(2)}+\frac{1}{\zeta^{2}(2)}\left(2 \gamma-1-2 \frac{\zeta^{\prime}(2)}{\zeta(2)}\right) x+\sum_{d \leq x} \frac{\mu * \mu(d)}{d} \Delta\left(\frac{x}{d}\right)+O(\kappa(x)),
\end{aligned}
$$


which completes the proof of Eq. (26). By using the fact that $\frac{\mu * \phi}{\mathrm{id}} * \mathrm{id}_{-2 m}=$ $\frac{\mu * \mu}{\mathrm{id}} * \sigma_{-2 m}$, together with Eqs. (6), (16), and (18) we get

$$
\begin{aligned}
\sum_{d \ell \leq x} \frac{\mu * \phi(d)}{d} \frac{1}{\ell^{2 m}} & =\sum_{d \leq x} \frac{\mu * \mu(d)}{d} \sum_{\ell \leq x / d} \sigma_{-2 m}(\ell) \\
& =\frac{\zeta(1+2 m)}{\zeta^{2}(2)} x+\sum_{d \leq x} \frac{\mu * \mu(d)}{d} \Delta_{-2 m}\left(\frac{x}{d}\right)+O_{m}(\kappa(x)) .
\end{aligned}
$$

Therefore, Eq. (27) is proved.

Lemma 8. For any large positive number $x>5$, we have

$$
\begin{aligned}
\sum_{n \leq x} \sum_{d \mid n} \frac{\mu * \psi(d)}{d} & =\frac{1}{\zeta(4)} x \log x+\frac{1}{\zeta(4)}\left(2 \gamma-1-2 \frac{\zeta^{\prime}(4)}{\zeta(4)}\right) x \\
& +\sum_{d \leq x} \frac{|\mu| * \mu(d)}{d} \Delta\left(\frac{x}{d}\right)+O\left(\frac{\delta(x)}{x^{1 / 2}}\right),
\end{aligned}
$$

and

$$
\begin{aligned}
\sum_{d \ell \leq x} \frac{\mu * \psi(d)}{d} \frac{1}{\ell^{2 m}}= & \frac{\zeta(1+2 m)}{\zeta(4)} x \\
& +\sum_{d \leq x} \frac{|\mu| * \mu(d)}{d} \Delta_{-2 m}\left(\frac{x}{d}\right)-\frac{\zeta(2 m)}{2 \zeta(2)}+O_{m}\left(\frac{\delta(x)}{x^{1 / 2}}\right)
\end{aligned}
$$

for any positive integer $m$.

Proof. From the identity $\frac{\mu * \psi}{\mathrm{id}} * \mathbf{1}=\frac{\mu *|\mu|}{\mathrm{id}} * \tau$, we have

$$
\sum_{k \leq x} \sum_{d \mid k} \frac{\mu * \psi(d)}{d}=\sum_{d \leq x} \frac{\mu *|\mu|(d)}{d} \sum_{\ell \leq x / d} \tau(\ell) .
$$

Using Eqs. (5), (19) and (20), we obtain the formula Eq. (28). Now, we use the identity $\frac{\mu * \psi}{\mathrm{id}} * \mathrm{id}_{-2 m}=\frac{\mu *|\mu|}{\mathrm{id}} * \sigma_{-2 m}$ to write our second sums as follows

$$
\sum_{d \ell \leq x} \frac{\mu * \psi(d)}{d} \frac{1}{\ell^{2 m}}=\sum_{d \leq x} \frac{\mu *|\mu|(d)}{d} \sum_{\ell \leq x / d} \sigma_{-2 m}(\ell) .
$$

Again, we use Eq. (6) to get

$$
\sum_{d \ell \leq x} \frac{\mu * \psi(d)}{d} \frac{1}{\ell^{2 m}}=\sum_{d \leq x} \frac{\mu *|\mu|(d)}{d}\left(\zeta(1+2 m) \frac{x}{d}-\frac{1}{2} \zeta(2 m)+\Delta_{-2 m}\left(\frac{x}{d}\right)\right) .
$$

Applying Eqs. (19) and (21) to the above, we deduce the desired result.

Now we are ready to prove our main theorems. 


\subsection{Proofs of the Theorems}

Proof of Theorem 1. First, we take $f=$ id into Eq. (1) to get

$$
\begin{aligned}
M_{r}(x ; \mathrm{id})= & \frac{1}{2} \sum_{n \leq x} 1+\frac{1}{r+1} \sum_{d \ell \leq x} \frac{\mu * \mathrm{id}(d)}{d} \\
& +\frac{1}{r+1} \sum_{m=1}^{[r / 2]}\left(\begin{array}{c}
r+1 \\
2 m
\end{array}\right) B_{2 m} \sum_{d \ell \leq x} \frac{\mu * \mathrm{id}(d)}{d} \frac{1}{\ell^{2 m}} \\
= & \frac{1}{2} \sum_{n \leq x} 1+\frac{1}{r+1} \sum_{n \leq x} \sum_{d \mid n} \frac{\phi(d)}{d} \\
& +\frac{1}{r+1} \sum_{m=1}^{[r / 2]}\left(\begin{array}{c}
r+1 \\
2 m
\end{array}\right) B_{2 m} \sum_{d \ell \leq x} \frac{\phi(d)}{d} \frac{1}{\ell^{2 m}} .
\end{aligned}
$$

Applying Eqs. (22) and (23) above yields

$$
\begin{aligned}
K_{r}(x)= & \frac{1}{r+1} \sum_{d \leq x} \frac{\mu(d)}{d} \Delta\left(\frac{x}{d}\right) \\
& +\frac{1}{r+1} \sum_{d \leq x} \frac{\mu(d)}{d} \sum_{m=1}^{[r / 2]}\left(\begin{array}{c}
r+1 \\
2 m
\end{array}\right) B_{2 m} \Delta_{-2 m}\left(\frac{x}{d}\right)+O_{r}(\delta(x)),
\end{aligned}
$$

which gives the desired result. We take $f=\phi$ into Eq. (1) to get

$$
\begin{aligned}
M_{r}(x ; \phi)= & \frac{1}{2} \sum_{n \leq x} \frac{\phi(n)}{n}+\frac{1}{r+1} \sum_{n \leq x} \sum_{d \mid n} \frac{\mu * \phi(d)}{d} \\
& +\frac{1}{r+1} \sum_{m=1}^{[r / 2]}\left(\begin{array}{c}
r+1 \\
2 m
\end{array}\right) B_{2 m} \sum_{d \ell \leq x} \frac{\mu * \phi(d)}{d} \frac{1}{\ell^{2 m}} .
\end{aligned}
$$

Using Lemma 2, as well as Eqs. (26) and (27), we get

$$
\begin{aligned}
L_{r}(x)= & \frac{1}{r+1} \sum_{n \leq x} \frac{\mu * \mu(n)}{n} \Delta\left(\frac{x}{n}\right) \\
& +\frac{1}{r+1} \sum_{n \leq x} \frac{\mu * \mu(n)}{n} \sum_{m=1}^{[r / 2]}\left(\begin{array}{c}
r+1 \\
2 m
\end{array}\right) B_{2 m} \Delta_{-2 m}\left(\frac{x}{n}\right) \\
& +O_{r}\left((\log x)^{2 / 3}(\log \log x)^{1 / 3}\right),
\end{aligned}
$$


as desired. Taking $f=\psi$ into Eq. (1) we get

$$
\begin{aligned}
M_{r}(x ; \psi)= & \frac{1}{2} \sum_{n \leq x} \frac{\psi(n)}{n}+\frac{1}{r+1} \sum_{d \ell \leq x} \frac{\mu * \psi(d)}{d} \\
& +\frac{1}{r+1} \sum_{m=1}^{[r / 2]}\left(\begin{array}{c}
r+1 \\
2 m
\end{array}\right) B_{2 m} \sum_{d \ell \leq x} \frac{\mu * \psi(d)}{d} \frac{1}{\ell^{2 m}} .
\end{aligned}
$$

Applying Lemma 3, as well as Eqs. (28) and (29) in the above formula yields

$$
\begin{aligned}
U_{r}(x)= & \frac{1}{r+1} \sum_{n \leq x} \frac{\mu *|\mu|(n)}{n} \Delta\left(\frac{x}{n}\right) \\
& +\frac{1}{r+1} \sum_{n \leq x} \frac{\mu *|\mu|(n)}{n} \sum_{m=1}^{[r / 2]}\left(\begin{array}{c}
r+1 \\
2 m
\end{array}\right) B_{2 m} \Delta_{-2 m}\left(\frac{x}{n}\right) \\
& -\frac{1}{4 \zeta(2)} \log x+O_{r}\left((\log x)^{2 / 3}\right) .
\end{aligned}
$$

This completes the proof of Theorem 1.

Proof of Theorem 2. By assuming the Riemann Hypothesis, and applying Eqs. (24) and (25) in Eq. (30), we immediately deduce that

$$
\begin{aligned}
K_{r}(x)= & \frac{1}{r+1} \sum_{d \leq x} \frac{\mu(d)}{d} \Delta\left(\frac{x}{d}\right) \\
& +\frac{1}{r+1} \sum_{d \leq x} \frac{\mu(d)}{d} \sum_{m=1}^{[r / 2]}\left(\begin{array}{c}
r+1 \\
2 m
\end{array}\right) B_{2 m} \Delta_{-2 m}\left(\frac{x}{d}\right)+O_{r}\left(\frac{\eta(x) \log x}{x^{1 / 2}}\right),
\end{aligned}
$$

which completes the proof of Theorem 2 .

\section{Proofs of Theorems 3 and 4}

To prove Theorems 3 we just need the following lemma.

Lemma 9. Under the hypotheses of Theorem 3, we have

$$
\begin{aligned}
\sum_{n \leq x} \frac{\mu(n)}{n^{2}}= & \frac{1}{\zeta(2)}+\sum_{|\beta| \leq T_{*}} \frac{x^{\rho-2}}{(\rho-2) \zeta^{\prime}(\rho)}+\frac{\pi^{2}}{\zeta(3)} x^{-4}+O\left(x^{-5}\right), \\
\sum_{n \leq x} \frac{\mu(n)}{n^{2}} \log \frac{x}{n}= & \frac{1}{\zeta(2)}\left(\log x-\frac{\zeta^{\prime}(2)}{\zeta(2)}\right) \\
& +\sum_{|\beta| \leq T_{*}} \frac{x^{\rho-2}}{(\rho-2)^{2} \zeta^{\prime}(\rho)}-\frac{\pi^{2}}{4 \zeta(3)} x^{-4}+O\left(x^{-5}\right),
\end{aligned}
$$


and

$$
\sum_{n \leq x} \frac{\mu(n)}{n}=\sum_{|\beta| \leq T_{*}} \frac{x^{\rho-1}}{(\rho-1) \zeta^{\prime}(\rho)}+\frac{4 \pi^{2}}{3 \zeta(3)} x^{-3}+O\left(x^{-5}\right) .
$$

Proof. The proof of the lemma can be found in [8, Lemma 3.5].

Proof of Theorem 3. We recall that

$$
\begin{aligned}
M_{r}(x ; \mathrm{id})= & \frac{1}{2} \sum_{n \leq x} 1+\frac{1}{r+1} \sum_{d \ell \leq x} \frac{\mu * \mathrm{id}(\mathrm{d})}{d} \\
& +\frac{1}{r+1} \sum_{m=1}^{[r / 2]}\left(\begin{array}{c}
r+1 \\
2 m
\end{array}\right) B_{2 m} \sum_{d \ell \leq x} \frac{\mu * \mathrm{id}(\mathrm{d})}{d} \frac{1}{\ell^{2 m}} .
\end{aligned}
$$

Using the fact that

$$
\frac{\mu * \mathrm{id}}{\mathrm{id}} * \mathbf{1}=\frac{\mu}{\mathrm{id}} * \tau, \quad \frac{\mu * \mathrm{id}}{\mathrm{id}} * \mathrm{id}_{-2 m}=\frac{\mu}{\mathrm{id}} * \sigma_{-2 m},
$$

and Eqs. (5) and (6), we get

$$
\begin{aligned}
M_{r}(x ; \text { id })= & \frac{[x]}{2}+\frac{x}{r+1} \sum_{n \leq x} \frac{\mu(n)}{n^{2}} \log \frac{x}{n}+\frac{x}{r+1}\left(2 \gamma-1+C_{\text {odd }}(r)\right) \sum_{n \leq x} \frac{\mu(n)}{n^{2}} \\
& -\frac{C_{\text {even }}(r)}{2(r+1)} \sum_{n \leq x} \frac{\mu(n)}{n}+\frac{1}{r+1} \sum_{n \leq x} \frac{\mu(n)}{n} \Delta\left(\frac{x}{n}\right) \\
& +\frac{1}{r+1} \sum_{m=1}^{[r / 2]}\left(\begin{array}{c}
r+1 \\
2 m
\end{array}\right) B_{2 m} \sum_{n \leq x} \frac{\mu(n)}{n} \Delta_{-2 m}\left(\frac{x}{n}\right) .
\end{aligned}
$$

Under the hypotheses of the theorem, we use Lemma 9 to obtain

$$
\begin{aligned}
M_{r}(x ; \mathrm{id})= & \frac{[x]}{2}+\frac{x \log x}{(r+1) \zeta(2)}+\frac{x}{(r+1) \zeta(2)}\left(2 \gamma-1-\frac{\zeta^{\prime}(2)}{\zeta(2)}+C_{\text {odd }}(r)\right) \\
& +\frac{1}{r+1} \sum_{n \leq x} \frac{\mu(n)}{n} \Delta\left(\frac{x}{n}\right)+\frac{1}{r+1} \sum_{m=1}^{[r / 2]}\left(\begin{array}{c}
r+1 \\
2 m
\end{array}\right) \\
& B_{2 m} \zeta(2 m) \sum_{n \leq x} \frac{\mu(n)}{n} \Delta_{-2 m}\left(\frac{x}{n}\right) \\
& +\frac{1}{r+1}\left(2 \gamma-1+C_{\text {odd }}(r)\right) \sum_{|\beta| \leq T_{*}} \frac{x^{\rho-1}}{(\rho-2) \zeta^{\prime}(\rho)} \\
& +\frac{1}{r+1} \sum_{|\beta| \leq T_{*}} \frac{x^{\rho-1}}{(\rho-2)^{2} \zeta^{\prime}(\rho)}-\frac{C_{\text {even }}(r)}{2(r+1)} \\
& \sum_{|\beta| \leq T_{*}} \frac{x^{\rho-1}}{(\rho-1) \zeta^{\prime}(\rho)}+O_{r}\left(x^{-3}\right)
\end{aligned}
$$


which completes the proof.

Proof of Theorem 4. To prove our theorem it suffices to show that

$$
\sum_{|\beta| \leq T_{*}} \frac{x^{-\frac{1}{2}+i \beta}}{(-j+i \beta) \zeta^{\prime}\left(\frac{1}{2}+i \beta\right)}=O\left(x^{-1 / 2}(\log x)^{5 / 4}\right)
$$

with $j=1 / 2$ and $3 / 2$. We take $\lambda=-1 / 2$ into Eq. (9), then $J_{-1 / 2}\left(T_{*}\right) \ll$ $T_{*}\left(\log T_{*}\right)^{1 / 4}$. Using the above and partial summation we have

$$
\sum_{|\beta| \leq T_{*}} \frac{1}{\beta\left|\zeta^{\prime}\left(\frac{1}{2}+i \beta\right)\right|} \ll\left[\frac{J_{-1 / 2}(t)}{t}\right]_{14}^{T_{*}}+\int_{14}^{T_{*}} \frac{J_{-1 / 2}(t)}{t^{2}} d t \ll\left(\log T_{*}\right)^{5 / 4},
$$

and the proof is complete.

Funding Open access funding provided by Johannes Kepler University Linz.

Open Access. This article is licensed under a Creative Commons Attribution 4.0 International License, which permits use, sharing, adaptation, distribution and reproduction in any medium or format, as long as you give appropriate credit to the original author(s) and the source, provide a link to the Creative Commons licence, and indicate if changes were made. The images or other third party material in this article are included in the article's Creative Commons licence, unless indicated otherwise in a credit line to the material. If material is not included in the article's Creative Commons licence and your intended use is not permitted by statutory regulation or exceeds the permitted use, you will need to obtain permission directly from the copyright holder. To view a copy of this licence, visit http://creativecommons. org/licenses/by/4.0/.

\section{References}

[1] Apostol, T.M.: Introduction to Analytic Number Theory. Springer, Berlin (1976)

[2] Balazard, M., de Roton, A.: Notes de lecture de l'article "Partial sums of the Möbius function" de Kannan Soundararajan. arXiv:0810.3587v1 [math.NT] (2008)

[3] Bordellés, O.: The composition of the GCD and certain arithmetic functions. J. Integer Sequences 13, Article 10.7.1 (2010)

[4] Broughan, K.A.: The average order of the Dirichlet series of the GCD-sum function. J. Integer Sequences 10, Article 07.4.2 (2007)

[5] Gonek, S.M.: On negative moments of the Riemann zeta-function. Mathematika 36, 71-88 (1989)

[6] Hejhal, D.: On the distribution of $\log \left|\zeta^{\prime}\left(\frac{1}{2}+i t\right)\right|$. In: Aubert, K.E., Bombieri, E., Goldfeld, D. (eds.) Number theory, trace formula and discrete groups, Symposium in Honor of Atle Selberg, Oslo, Norway, vol. 1989, pp. 343-370 . Academic Press, San Diego (1987) 
[7] Huxley, M.N.: Exponential sums and lattice points III. Proc. Lond. Math. Soc. 87, 591-609 (2003)

[8] Inoue, S., Kiuchi, I.: On sums of GCD-sum functions. Preprint

[9] Jia, R.Q.: Estimation of partial sums of series $\sum \mu(n) / n$. Kexue Tongbao. 30, 575-578 (1985)

[10] Kiuchi, I.: On sums of averages of generalized Ramanujan sums. Tokyo J. Math. 40, 255-275 (2017)

[11] Kiuchi, I.: Sums of averages of GCD-sum functions. J. Number Theory 176, 449-472 (2017)

[12] Kiuchi, I.: Sums of averages of generalized Ramanujan sums. J. Number Theory 180, 310-348 (2017)

[13] Kiuchi, I., Saad Eddin, S.: Sums of weighted averages of GCD-sum functions. Int. J. Number Theory 14, 2699-2728 (2018)

[14] Liu, H.-Q.: On Euler's function. Proc. R. Soc. Edinb. 146, 769-775 (2016)

[15] Maier, H., Montgomery, H.L.: On the sum of the Möbius function. Bull. Lond. Math. Soc. 41, 213-226 (2009)

[16] Sitaramachandra Rao, R., Suryanarayana, D.: The number of pairs of integers with L. C. M. $\leq x$. Arch. Math. (Basel) 21, 490-497 (1970)

[17] Soundararajan, K.: Partial sums of the Möbius function. J. Reine Angew. Math. 631, 141-152 (2009)

[18] Tanigawa, Y., Zhai, W.: On the GCD-sum functions. J. Integer Sequences 11, Article 08.2.3 (2008)

[19] Tóth, L.: A survey of GCD-sum functions. J. Integer Sequences. 13, Article 10.8.1 (2010)

[20] Walfisz, A.: Weylsche Exponentialsummen in der Neueren Zahlentheorie. Veb Deutscher Verlag Der Wissenschaften, Berlin (1963)

Lisa Kaltenböck and Sumaia Saad Eddin

Institute of Financial Mathematics and Applied Number Theory

Johannes Kepler University

Altenbergerstrasse 69

4040 Linz

Austria

e-mail: lisa.kaltenboeck@jku .at;

sumaia.saad_eddin@jku.at

Isao Kiuchi and Masaaki Ueda

Department of Mathematical Sciences, Faculty of Science

Yamaguchi University

Yoshida 1677-1

Yamaguchi 753-8512

Japan

e-mail: kiuchi@yamaguchi-u.ac.jp;

i001wb@yamaguchi-u.ac.jp 
Received: June 17, 2020.

Accepted: February 2, 2021.

Publisher's Note Springer Nature remains neutral with regard to jurisdictional claims in published maps and institutional affiliations. 